\title{
Est-il vraiment pertinent d'évaluer la rédaction du résumé dans le cadre de l'épreuve de lecture critique d'article scientifique? Réponse
}

\author{
Is it really relevant to assess the written summary aptitude \\ for the scientific article critical reading test? \\ Reply
}

Monsieur,

Nous remercions Hervé Maisonneuve pour l'intérêt porté à notre article. Le débat qu'il aborde dépasse largement notre travail d'évaluation de la correction du résumé, puisqu'il remet tout simplement en cause l'idée même de demander aux étudiants de rédiger un résumé dans le cadre de l'épreuve de lecture critique d'article scientifique lors des épreuves classantes nationales.

Tout d'abord, nous partageons pleinement l'avis d'Hervé Maisonneuve quant au caractère indispensable à la formation des étudiants de l'analyse d'articles médicaux. La méthode choisie (questions + résumé) pour l'évaluation de l'acquisition de cette capacité ne parait pas optimale. Cependant, puisque cette méthode d'évaluation est actuellement celle de référence, nous sommes dans l'obligation de préparer au mieux nos étudiants pour cette épreuve.

Nous partageons aussi son avis sur le fait que la rédaction du résumé laisse peu de place à l'imagination. Cependant l'imagination a-t-elle une place dans la lecture critique d'article ou encore en médecine quotidienne de soin? Il s'agit, dans cette épreuve, simplement, comme il le rapporte, de prendre les phrases aux bons endroits dans l'article à examiner et de les rapporter en bonne place dans le résumé. Notre expérience, beaucoup moins grande que la sienne, mais reposant essentiellement sur l'apprentissage du résumé des articles médicaux nous fait dire que la réalisation du résumé n'est pas aussi simple et facile pour tous nos étudiants. En effet, lorsque nous demandons aux étudiants d'élaborer un résumé après avoir analysé l'article pour répondre à différentes questions d'ordre méthodologique, celui-ci est le plus souvent correctement rédigé. Par contre, lorsque les mêmes étudiants rédigent un résumé à partir d'un article sur lequel ils n'ont eu aucune question et qu'ils n'ont pas analysé, les résultats sont nettement moins bons. Pourquoi ? Parce que l'acquisition de la «recette»proposée (c'est celle qu' on leur apprend) ne peut s'obtenir que par la pratique. Et nous savons très bien que la pratique de cet exercice ne sera faite par les étudiants que si elle est sanctionnée par un examen noté. Nous avons débuté cette année au sein des facultés de médecine de Toulouse, après avoir pris conscience de cela, une conférence préparatoire dédiée uniquement à la réalisation du résumé. Nos premières expériences montrent qu'il s'agit d'une réelle nécessité. 
Quant à la question concernant le point de savoir s'il est utile que les étudiants sachent faire un résumé d'article médical, notre réponse est clairement positive. Même si la grande majorité d'entre eux ne seront jamais universitaires ou ne rédigeront jamais d'articles médicaux, par contre ils recevront tous des visiteurs médicaux leur vantant les mérites supposés d'un nouveau médicament ou d'un dispositif médical et leur présentant des publications scientifiques à l'appui. Comment pourraient-ils se forger une opinion fiable s'ils ne peuvent pas rapidement en identifier les phrases importantes, ce qui revient à en élaborer un rapide résumé ?

Comme il l'évoque très bien, les résumés originaux ne sont pas toujours bien construits et comportent des données qui ne sont pas toujours bien situées. Ceci est d'autant plus dommageable que nos enseignements insistent sur l'importance de la juste place des mots et des idées. On demande de noter les résumés en fonction du résumé type rédigé par les auteurs et ceci malgré ses inévitables imperfections. On peut raisonnablement penser que celui choisi pour l'examen de lecture critique d'article sera bien construit. Tout ceci participe à la difficulté de l'enseignement et de la correction de l'épreuve de rédaction du résumé.

Finalement, il parait important de consacrer du temps à l'apprentissage de la rédaction du résumé et surtout à sa correction. Plus nous y réfléchissons et plus nous prenons conscience de la demande des étudiants. La rédaction du résumé est la seule épreuve faisant appel à un esprit de synthèse et non à une mémorisation poussée... mais ceci est un autre débat.

Enfin, en conclusion, pourquoi ce qui est bon pour les étudiants des grandes écoles (exercices de contraction de textes), ne le serait-il pas pour les étudiants en médecine?

Fabrice Muscari Jean-Louis Montastruc Gilles Fourtanier

Service de chirurgie digestive

CHU Rangueil, 1 avenue J. Poulhès, TSA 50032, 31059 Toulouse Cedex 9, France Mailto : muscari.f@ chu-toulouse.fr 